\title{
The compartmental approach to revision of partial knee arthroplasty results in nearer-normal gait and improved patient reported outcomes compared to total knee arthroplasty
}

\author{
Amy J. Garner ${ }^{1,2,3}$ (D) . Oliver W. Dandridge ${ }^{4} \mathbb{D} \cdot{\text { Richard J. van } \text { Arkel }^{4}(\mathbb{D} \cdot \text { Justin P. Cobb }}^{1}$ (D)
}

Received: 30 March 2021 / Accepted: 30 July 2021 / Published online: 20 August 2021

(c) The Author(s) 2021

\begin{abstract}
Purpose This study investigated the gait and patient reported outcome measures of subjects converted from a partial knee arthroplasty to combined partial knee arthroplasty, using a compartmental approach. Healthy subjects and primary total knee arthroplasty patients were used as control groups.

Methods Twenty-three patients converted from partial to combined partial knee arthroplasty were measured on the instrumented treadmill at top walking speeds, using standard gait metrics. Data were compared to healthy controls $(n=22)$ and primary posterior cruciate-retaining total knee arthroplasty subjects $(n=23)$ where surgery were performed for one or twocompartment osteoarthritis. Groups were matched for age, sex and body mass index. At the time of gait analysis, combined partial knee arthroplasty subjects were median 17 months post-revision surgery (range 4-81 months) while the total knee arthroplasty group was median 16 months post-surgery (range 6-150 months). Oxford Knee Scores and EuroQol-5D 5L scores were recorded at the time of treadmill assessment, and results analysed by question and domain.

Results Subjects revised from partial to combined partial knee arthroplasty walked $16 \%$ faster than total knee arthroplasty (mean top walking speed $6.4 \pm 0.8 \mathrm{~km} / \mathrm{h}$, vs. $5.5 \pm 0.7 \mathrm{~km} / \mathrm{h} p=0.003$ ), demonstrating nearer-normal weight-acceptance rate $(p<0.001)$, maximum weight-acceptance force $(p<0.006)$, mid-stance force $(p<0.03)$, contact time $(p<0.02)$, double support time $(p<0.009)$, step length $(p=0.003)$ and stride length $(p=0.051)$ compared to primary total knee arthroplasty. Combined partial knee arthroplasty subjects had a median Oxford Knee Score of 43 (interquartile range 39-47) vs. 38 (interquartile range 32-41, $<<0.02$ ) and reported a median EQ-5D 0.94 (interquartile range 0.87-1.0) vs. 0.84 (interquartile range $0.80-0.89, p=0.006$ ).
\end{abstract}

Conclusion This study finds that a compartmental approach to native compartment degeneration following partial knee arthroplasty results in nearer-normal gait and improved patient satisfaction compared to total knee arthroplasty.

Level of evidence III.

Keywords Multicompartmental arthroplasty $\cdot$ Compartmental arthroplasty $\cdot$ Bicompartmental $\cdot$ Bi-unicondylar $\cdot$ Gait . Satisfaction

Amy J. Garner

a.garner@imperial.ac.uk

Oliver W. Dandridge

o.dandridge18@imperial.ac.uk

Richard J. van Arkel

r.vanarkel@imperial.ac.uk

Justin P. Cobb

j.cobb@imperial.ac.uk

1 Imperial College London, MSk Lab, Sir Michael Uren Biomedical Engineering Research Hub, Imperial

College London, White City Campus, 80-92 Wood Lane,

London W12 0BZ, UK
2 Royal College of Surgeons of England and Dunhill Medical Trust Clinical Research Fellowship, Royal College of Surgeons of England, 35-43 Lincoln's Inn Fields, London WC2A 3PE, UK

3 Health Education Kent, Surrey and Sussex, Stewart House, 32 Russell Square, London WC1B 5DN, UK

4 Biomechanics Group, Department of Mechanical Engineering, Imperial College London, City \& Guilds Building, Exhibition Road, London SW7 9AG, UK 


\section{Introduction}

Partial knee arthroplasty (PKA) may be appropriate for up to $50 \%$ of primary knee arthroplasty cases [28] but currently accounts for just $10 \%$ of procedures in large joint replacement registries $[1,26]$. In part, the low usage rate of PKA is attributed to the significantly higher revision rates compared to total knee arthroplasty (TKA) [1,26], though just $17 \%$ of patients have tricompartmental disease [38]. At 13 years, cumulative revision rates of cemented unicompartmental arthroplasty (UKA) are $14.9 \%$, while for primary patellofemoral arthroplasty (PFA) it is $24.4 \%$, compared to $4.18 \%$ for the TKA over the same time frame [1].

Progression of osteoarthritis remains the most common reason for revision of PKA [1, 22, 37] and most often involves the implantation of a standard primary TKA implant. In some instances, PKA removal can result in significant bone loss, necessitating the use of stems, metaphyseal augments or implants with increased constraint, potentially reducing post-revision patient satisfaction and function $[22,29,37]$. An alternative to revision to TKA is the addition of a second PKA to treat a newly symptomatic native compartment. This 'compartmental approach' converts PKA to a combined partial knee arthroplasty (CPKA) without the need for removal of a well-functioning primary PKA [18], with some evidence of successful outcomes in the medium $[2,7,8]$ and longer term $[19,35]$. CPKA have been classified according to the combination of PKA used and may be bicompartmental (BCA), that is the combination of PFA with either a medial (BCA-M) or lateral (BCA-L) UKA, or bi-unicondylar (Bi-UKA), the ipsilateral combination of medial and lateral UKA $[14,18]$ (Fig. 1.)

The functional outcomes and patient reported outcome metrics (PROMs) associated with CPKA in the staged setting (Fig. 2) are not known [12]. This study aimed to compare the post-operative gait and patient reported outcomes of staged CPKA to those of healthy controls and primary

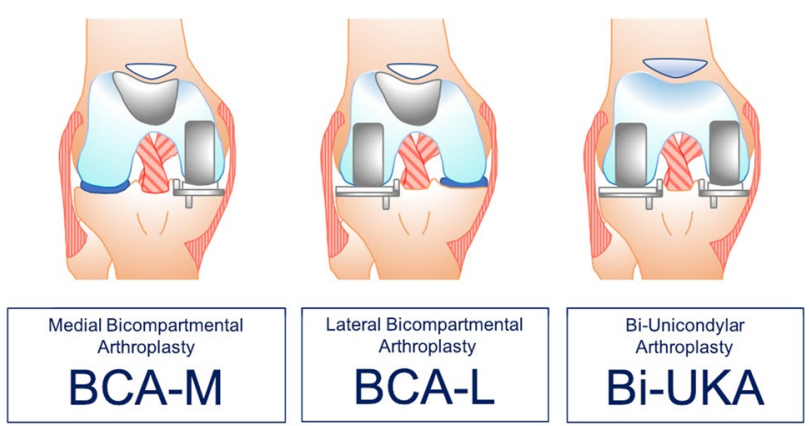

Fig. 1 Classification of combined partial knee arthroplasty. Adapted from Garner et al. [14]

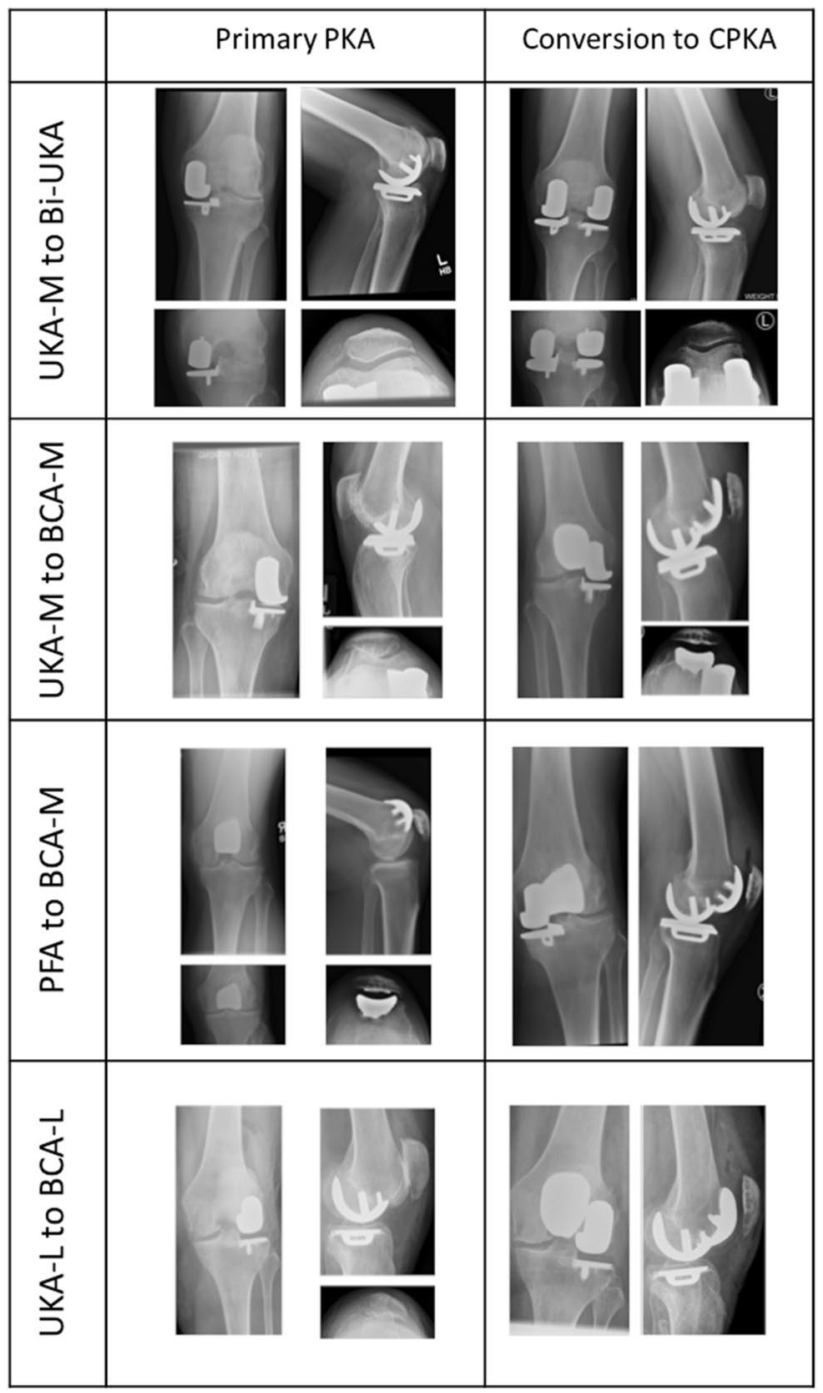

Fig. 2 Radiographic examples of partial knee arthroplasty (PKA) procedures revised to combined partial knee arthroplasty (CPKA) for native compartment degeneration, using a compartmental approach. Medial unicompartmental arthroplasty (UKA-M), lateral unicompartmental arthroplasty (UKA-L), patellofemoral arthroplasty (PFA), medial bicompartmental arthroplasty (BCA-M), lateral bicompartmental arthroplasty (BCA-L), bi-unicondylar arthroplasty (Bi-UKA)

TKA subjects. The null hypothesis was that there would be no difference between staged CPKA and primary TKA in terms of gait and PROMs.

\section{Patients and methods}

\section{The compartmental approach}

Primary PKA had been performed for single compartment OA. The indications for conversion to CPKA are outlined in Table 1, primarily for degeneration of one of the remaining 
Table 1 Indications and contra-indications for conversion of PKA to CPKA

\begin{tabular}{|c|c|}
\hline Indications for conversion to CPKA & Contra-indications \\
\hline Osteoarthritic degeneration of a single native compartment & Osteoarthritic degeneration of two-native compartments \\
\hline Well-functioning primary partial knee arthroplasty in situ ${ }^{a}$ & Loose/unstable/problematic primary partial knee arthroplasty \\
\hline Functional anterior cruciate ligament ${ }^{\mathrm{b}}$ & Anterior cruciate ligament dysfunction ${ }^{\mathrm{b}}$ \\
\hline Correctable varus/valgus & Inflammatory arthropathy \\
\hline Medically high risk for revision surgery & Evidence of periprosthetic infection \\
\hline
\end{tabular}

${ }^{\mathrm{a}} \mathrm{A}$ worn polyethylene bearing may be exchanged at the time of second surgery and is not considered a contraindication

${ }^{\mathrm{b}} \mathrm{ACL}$ dysfunction in the elderly is a relative contraindication, provided that the knee is otherwise stable

compartments, where the third compartment was intact and disease-free on plain radiographs, and the ACL functional on clinical examination and radiographic imaging $(<7 \mathrm{~mm}$ anterior tibial translation [9]). In the practise of the senior author, the absence of a functional ACL is a relative contraindication to conversion to CPKA, permissible in the elderly, where overall laxity of the joint is reduced, and the risk of major revision surgery from PKA to TKA outweighs the possibility of reduced anterior-posterior stability with CPKA. Inflammatory arthropathy is considered an absolute contraindication.

Pre-operative imaging included plain radiographs of the knee, specifically the anterior-posterior, lateral, skyline and Rosenberg views. Use of cross-sectional imagine including magnetic resonance imaging was not routinely used. Procedures were performed using conventional instrumentation. Where conversion to BCA occurred, the original approach was re-used and extended as required. Where conversion to Bi-UKA occurred, if the original incision was in the midline, it was re-opened, and then a new parapatellar arthrotomy used to approach the native compartment (for example, a lateral parapatellar approach would be used to approach the lateral compartment, even though the original procedure used a medial parapatellar approach to the medial compartment). If the original incision was medial or lateral to the midline, a new, parallel incision was made over the native compartment. There were no reported incidences of unplanned intraoperative conversion to TKA.

\section{Gait analysis}

A retrospective cohort study was conducted, based on the operative caseload of the senior author, from 2009 to 2019. All subjects converted from a PKA to CPKA were considered for the study. Forty-six CPKA patients were excluded (Fig. 3, Supplementary Table A), three patients declined to participate following telephone invitation, none of whom reported further ipsilateral knee surgery. Four patients were uncontactable via telephone or e-mail. Twenty-three CPKA subjects entered the study (Fig. 3). They consisted of staged BCA-M $(n=5,83 \%$ female $)$, staged BCA-L $(n=3,100 \%$ female) and staged Bi-UKA ( $n=15,27 \%$ female). Of them, eight had undergone primary PKA under the care of other surgeons, therefore, the combinations of implants varied (Supplementary Table B). Of the CPKA group, five subjects had undergone contralateral UKA-M and one had a contralateral UKA-L.

\section{Matching from the prospectively collected gait database}

For 9 years, with informed consent and ethical approval (Imperial College Healthcare NHS Trust, 10/H0807/101 and NRES Committee South Central, 136430) three highvolume senior surgeons have contributed arthroplasty patients for measurement on the instrumented treadmill (Kistler Gaitway, Kistler Instrument Corporation, Amherst, NY). Subject data, in addition to that of healthy volunteers, has been pooled to form a prospectively collected gait database. Healthy controls $(n=22)$ were identified from this database, matched for age $(p=0.2)$, sex $(p=1)$, body mass index $(p=0.4)$ and height $(p=0.8)$ to those of the CPKA group (Table 2, Fig. 3.)

\section{Matching to TKA subjects}

The design of this study was 'non-inferiority' to primary TKA. Forty-nine posterior cruciate-retaining TKA subjects were identified from the prospectively collected database (Fig. 3). TKA subjects were excluded if they were pre-revision $(n=5)$, had an ipsilateral hip replacement $(n=2)$, contralateral knee OA $(n=9)$ or unsuitable demographics after matching $(n=5)$. Of the remaining, pre-operative radiographs were assessed for Kellgren and Lawrence $(\mathrm{KL})$ grade per compartment and anterior tibial translation $>7 \mathrm{~mm}$, suggestive of ACL dysfunction [9]. TKA subjects $(n=5)$ were excluded if they had tricompartmental $\mathrm{OA}(\mathrm{KL} \geq 2)$ or radiographic evidence of anterior cruciate ligament (ACL) dysfunction. Twenty-three posterior cruciate-retaining TKA subjects entered this study, of whom 15 had KL $\geq 2$ OA confined to the medial $(n=12)$ or lateral $(n=3)$ compartment, with the remaining 8 subjects having two-compartment 


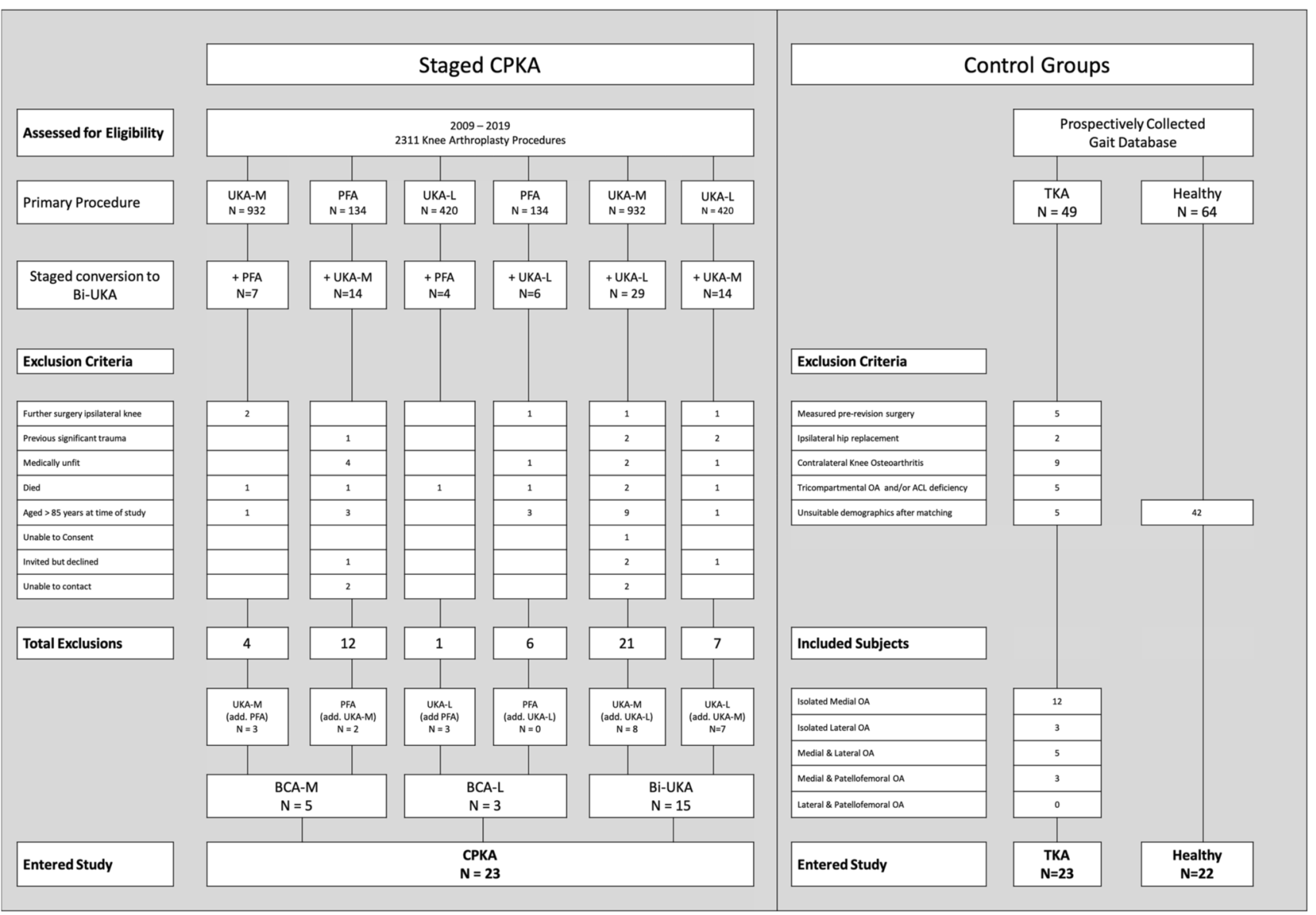

Fig. 3 STROBE diagram depicting the route of subject entry into the study. Number of subjects $(N=)$, partial knee arthroplasty (PKA), medial unicompartmental knee arthroplasty (UKA-M), lateral unicompartmental knee arthroplasty (UKA-L), patellofemoral arthro-

disease (medial and lateral $n=5$, medial and patellofemoral $n=3)$. TKA subjects were median 16 months post-surgery (range 6-150 months). All would have been eligible for PKA or CPKA under the senior author's practice.

\section{Treadmill testing}

With ethical approval from our institutional review board (Imperial College Healthcare NHS Trust 10/H0807/101 and NRES Committee South Central IRAS 136430) and informed consent, subjects were measured on the flat instrumented treadmill, by a research assistant blinded to the study group, following a 2 min acclimatisation 'warm up' at $3.5 \mathrm{~km} / \mathrm{h}$. The speed was then increased in $0.5 \mathrm{~km} / \mathrm{h}$ increments, dictated by the subject, until they reached their 'top walking speed' defined as the fastest speed at which they could walk comfortably or before they felt compelled to run. All subjects completed the test without the assistance of the hand safety rail. The vertical component of the ground plasty (PKA), medial bicompartmental knee arthroplasty (BCA-M), lateral bicompartmental knee arthroplasty (BCA-L), bi-unicondylar knee arthroplasty (Bi-UKA), combined partial knee arthroplasty (CPKA), posterior cruciate-retaining total knee arthroplasty (TKA)

reaction forces; temporospatial measurements and centre of pressure readings for both limbs were recorded by two tandem force plates, sampling at $100 \mathrm{~Hz}$ frequency over $10 \mathrm{~s}$, located beneath the moving belt. Data were normalised to account for differences in body mass (body mass/gravity) and leg length, using Hof scaling [20].

\section{Statistical analysis}

The difference in gait of medial UKA subjects compared to TKA subjects at top walking speeds formed the basis of a power calculation, resolving that 16 subjects per group would be required to detect gait differences at top walking speeds, with $80 \%$ power and $95 \%$ confidence [23]. Groups were matched for age, sex and body mass index in IBM ${ }^{\circledR}$ SPSS ${ }^{\circledR}$ version 27. Gait data were analysed with a custom Mathworks ${ }^{\circledR}$ MatLab $^{\circledR}$ script and analysed in IBM ${ }^{\circledR}$ SPSS $^{\circledR}$ version 27. The Shapiro-Wilk test indicated that normal distribution could not be assumed for a number of variables, consequently, data were compared using Kruskal-Wallis, 
Table 2 Demographics, gait characteristics at top walking speeds and patient reported outcomes of staged combined partial knee arthroplasty (CPKA) subjects compared to healthy controls and total knee arthroplasty (TKA) subjects

\begin{tabular}{llll}
\hline Subject & Healthy & Staged CPKA & TKA \\
\hline Number of knees (N=) & 22 & 23 & 23 \\
Sex: $M: F$ & $10: 12$ & $11: 12$ & $10: 13$ \\
Age (years) & $64 \pm 8$ & $68 \pm 10$ & $68 \pm 10$ \\
Body mass index (kg/m ${ }^{2}$ ) & $26.8 \pm 5.4$ & $28.4 \pm 5.4$ & $28.7 \pm 3.5$ \\
Height (cm) & $170 \pm 9$ & $172 \pm 6$ & $171 \pm 11$ \\
Mean months post-surgery (SD) & & $22 \pm 21^{\mathrm{c}}$ & $42 \pm 50^{\mathrm{c}}$ \\
Median months post-surgery (range) & & $17(4-81)$ & $16(6-150)$ \\
Top walking speed (km/h) & $7.2 \pm 0.7^{\mathrm{a}, \mathrm{b}}$ & $6.4 \pm 0.8^{\mathrm{a}, \mathrm{c}}$ & $5.5 \pm 0.7^{\mathrm{b}, \mathrm{c}}$ \\
Hof speed (H) & $0.73 \pm 0.1^{\mathrm{a}, \mathrm{b}}$ & $0.64 \pm 0.1^{\mathrm{a}, \mathrm{c}}$ & $0.55 \pm 0.1^{\mathrm{b}, \mathrm{c}}$ \\
Weight acceptance rate (BW/s) & $10.8 \pm 3.5^{\mathrm{b}}$ & $10.6 \pm 3.6^{\mathrm{c}}$ & $6.8 \pm 2.6^{\mathrm{b}, \mathrm{c}}$ \\
Maximum weight-acceptance force (BW) & $1.6 \pm 0.2^{\mathrm{a}, \mathrm{b}}$ & $1.4 \pm 0.2^{\mathrm{a}, \mathrm{c}}$ & $1.2 \pm 0.1^{\mathrm{b}, \mathrm{c}}$ \\
Mid-stance force (BW) & $0.5 \pm 0.1^{\mathrm{a}, \mathrm{b}}$ & $0.6 \pm 0.1^{\mathrm{a}, \mathrm{c}}$ & $0.7 \pm 0.1^{\mathrm{b}, \mathrm{c}}$ \\
Push-off force (BW) & $0.98 \pm 0.2$ & $0.96 \pm 0.1$ & $0.97 \pm 0.1$ \\
Push-off rate (BW/s) & $4.1 \pm 1.0$ & $4.0 \pm 0.9$ & $3.6 \pm 0.7$ \\
Step length (cm) & $82 \pm 8^{\mathrm{b}}$ & $79 \pm 7^{\mathrm{c}}$ & $70 \pm 7^{\mathrm{b}, \mathrm{c}}$ \\
Stride length (cm) & $165 \pm 16^{\mathrm{b}}$ & $156 \pm 14$ & $144 \pm 17^{\mathrm{b}}$ \\
Gait width (cm) & $12 \pm 3$ & $14 \pm 2$ & $13 \pm 4$ \\
Cadence (step/min) & $60 \pm 5^{\mathrm{b}}$ & $57 \pm 5$ & $53 \pm 5^{\mathrm{b}}$ \\
Impulse (BW/s) & $387 \pm 23$ & $378 \pm 22$ & $375 \pm 21$ \\
Double support time (s) & $0.28 \pm 0.1^{\mathrm{b}}$ & $0.29 \pm 0.1^{\mathrm{c}}$ & $0.36 \pm 0.1^{\mathrm{b}, \mathrm{c}}$ \\
Contact time (s) & $1.3 \pm 0.1^{\mathrm{b}}$ & $1.4 \pm 0.1^{\mathrm{c}}$ & $1.5 \pm 0.2^{\mathrm{b}, \mathrm{c}}$ \\
OKS mean (range) & & $41.3 \pm 7.4^{\mathrm{c}}$ & $37.1 \pm 5.1^{\mathrm{c}}$ \\
OKS median (interquartile range) & $43(39-47)$ & $38(32-41)$ \\
EQ-5D mean & & $0.91 \pm 0.1^{\mathrm{c}}$ & $0.83 \pm 0.1^{\mathrm{c}}$ \\
EQ-5D median (interquartile range) & $0.94(0.87-1.0)$ & $0.84(0.80-0.89)$ \\
\hline
\end{tabular}

All values are means with standard deviations unless otherwise stated. Demongraphics subject to ANOVA, gait variables subject to Kruskal-Wallis then Mann-Whitney test with Bonferroni correction

$B W$ normalised to body weight

All tests, significance $p$ value $<0.05$.

${ }^{\text {a}}$ Healthy vs. CPKA $<0.05$

${ }^{\mathrm{b}}$ Healthy vs. TKA $p<0.05$

${ }^{\mathrm{c}}$ CPKA vs. TKA $p<0.05$ then the Mann-Whitney test with Bonferroni correction $(\alpha=0.05)$.

While gait analysis were the primary outcome of this study, patient reported outcomes were collected as a secondary outcome, measured at the time of treadmill assessment using the Oxford Knee Score (OKS) and EuroQol (EQ-5D) scores, Average scores were subject to the Mann-Whitney test according to the overall score and by individual question/domain $(\alpha=0.05)$. CPKA subgroup data were descriptively analysed.

\section{Results}

\section{Functional analysis}

The CPKA group walked, on average, $16 \%$ faster than primary TKA, though neither arthroplasty group walked as fast as the healthy subjects ( $p<0.01$, Fig. 4, Table 2.) This remained the case after correcting for leg length. At top walking speeds, CPKA had near-normal weight-acceptance rate (CPKA vs. healthy $p=1$ ) whereas TKA weightacceptance was reduced compared to healthy $(p<0.001)$ and CPKA $(p<0.006)$ subjects (Table 2, Fig. 5). Both implant groups demonstrated reduced maximum weight-acceptance force compared to the healthy subjects, though TKA was associated with a greater reduction (all $<0.01$, Table 2, Fig. 5). Similarly, CPKA and TKA demonstrated higher mid-stance forces than healthy subjects $(p<0.001)$, but the 


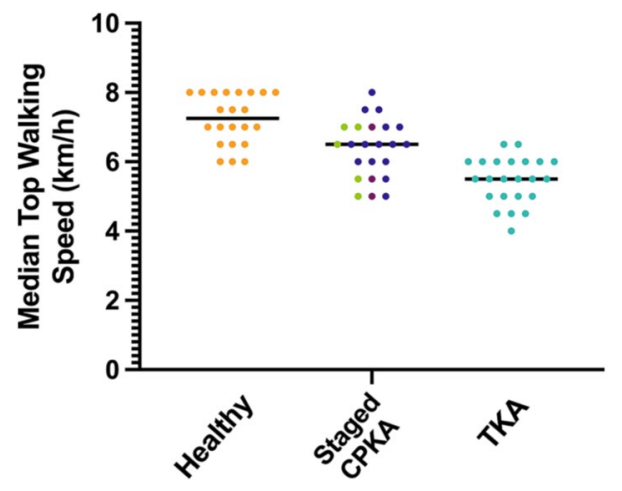

Fig. 4 Median top walking speed $(\mathrm{km} / \mathrm{h})$ for subjects in study 4: staged combined partial knee arthroplasty (staged CPKA) compared to healthy subjects and posterior cruciate-retaining total knee arthroplasty (TKA). Of the staged CPKA group, the individual procedures are depicted: staged medial bicompartmental arthroplasty (green), staged lateral bicompartmental arthroplasty (light purple), staged biunicondylar arthroplasty (dark purple)

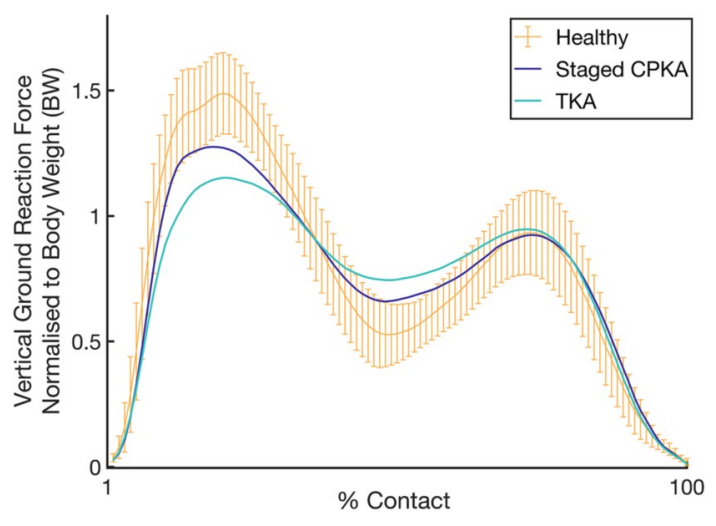

Fig. 5 Vertical ground reaction forces during stance phase of gait for subjects revised from a partial to combined partial knee arthroplasty (CPKA) compared to primary posterior cruciate-retaining total knee arthroplasty (TKA), normal range for healthy subjects shown with 95\% confidence intervals difference was smaller following CPKA $(p<0.03)$. CPKA had nearer-normal contact time (CPKA vs. TKA $p=0.012$ ) and double support time (CPKA vs. TKA $p<0.01)$.

Staged CPKA median step length were $78 \mathrm{~cm}$ (IQR $73-82 \mathrm{~cm}), 5 \%$ shorter than healthy controls, median $82 \mathrm{~cm}$ (IQR 77-88 cm), whilst TKA measured 15\% shorter step lengths, median $70 \mathrm{~cm}$ (IQR 66-74 cm, Table 2, Fig. 6). Differences in step length were replicated in stride length: staged CPKA median stride length were $156 \mathrm{~cm}$ (IQR 148-165), $8 \mathrm{~cm}$ shorter than healthy subjects, median $164 \mathrm{~cm}$ (IQR $155-178 \mathrm{~cm}$ ), but $12 \mathrm{~cm}$ longer than TKA, median $142 \mathrm{~cm}$ (IQR 133-157 cm, Table 2, Fig. 6).

\section{Patient reported outcomes}

Mean OKS in the CPKA group was $41.3 \pm 7.4$ compared to TKA $37.1 \pm 5.1(p<0.02$, Fig. 7 , Table 3$)$. CPKA scored equal to or higher than TKA in all questions (Q) of the OKS, reaching significance in questions related to use of transport (Q3), kneeling (Q7) and instability symptoms (Q10, all $p<0.03)$. Similarly, CPKA subjects reported a mean EQ-5D $0.91 \pm 0.1$ compared to TKA $0.83 \pm 0.2(p=0.006$, Fig. 7 , Table 4). CPKA scored equal or closer to 1 than TKA in all domains, reaching significance in mobility $(p=0.006)$, usual activities $(p=0.033)$ and pain $(p=0.033)$.

\section{Discussion}

The most important finding of this study is that, despite second surgery, patients converted to CPKA, for native compartment osteoarthritis, were found to have a more normal gait than matched high functioning primary TKA subjects, in whom, surgery had been performed for one or two-compartment disease with the reverse trend not identified for any of the measured metrics. The marked differences in top walking speed are particularly relevant, since life expectancy
Fig. 6 Median step length for all staged combined partial knee arthroplasty (staged CPKA) subjects compared to matched healthy subjects and posterior cruciate-retaining total knee arthroplasty subjects (TKA). Staged CPKA group, the individual procedures are depicted: staged medial bicompartmental arthroplasty (green), staged lateral bicompartmental arthroplasty (light purple), staged bi-unicondylar arthroplasty (dark purple)
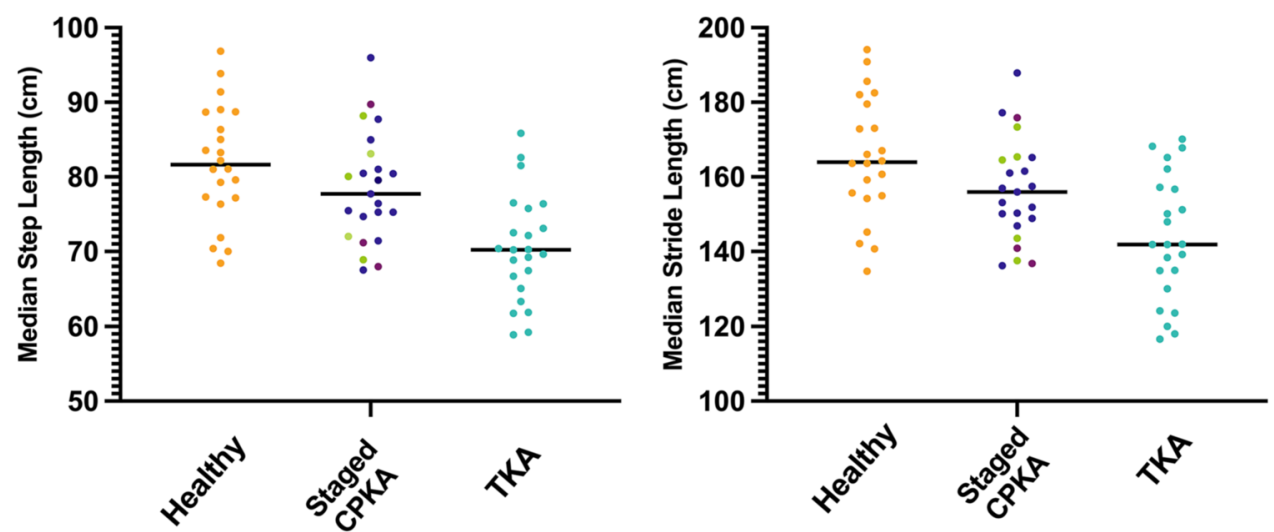

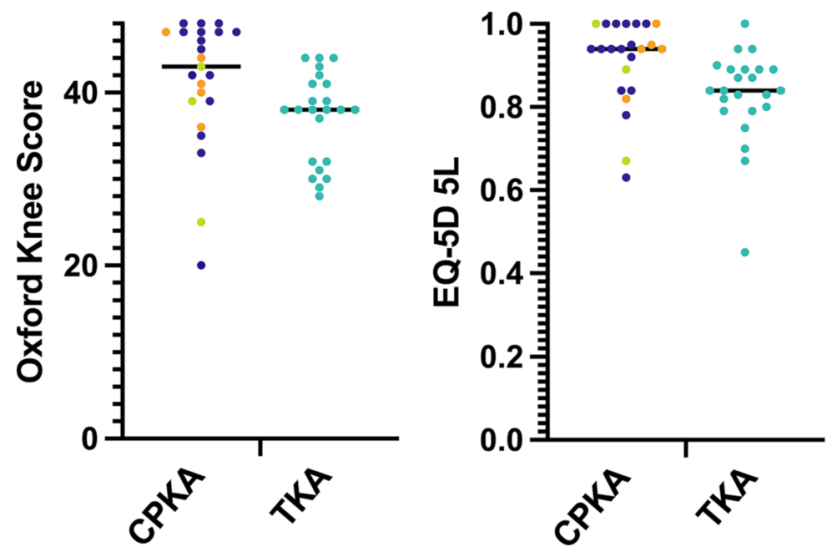

Fig. 7 Patient reported outcome metrics for combined partial knee arthroplasty (CPKA) compared to posterior cruciate-retaining total knee arthroplasty (TKA). Left, Median Oxford Knee Score, where 48 represents best possible function. Right: Median EuroQol-5D 5L, where a score of 1 represents best possible quality of life. Each point represents one study subject. CPKA subgroups are shown: BCA-M ( $n=5$, orange), BCA-L ( $n=3$, green), Bi-UKA ( $n=15$, purple)

improves with every $0.1 \mathrm{~m} / \mathrm{s}$ increase [39]. During weightacceptance and mid-stance, where the CPKA were significantly more normal than TKA, the quadriceps muscles are at their most active [30]. UKA and CPKA are significantly more anterior-posterior stable than TKA [15], and preserve the extensor efficiency of the knee, particularly at knee flexion angles associated with fast walking, whereas TKA is associated with a significant reduction in extensor efficiency of up to $43 \%$ at the same flexion angles [13]. These biomechanical differences may, in part, account for some of the observed differences in early stance.
Despite undergoing more than one operation, CPKA subjects reported good function on the OKS, corroborating the findings of others [34], with a good quality of life against EQ-5D. The OKS for TKA was in line with the TOPKAT study, a randomised control trial comparing primary UKA to TKA, suggesting that the TKA patients in this study performed as expected for a cohort of primary TKA [4]. However, while the overall scores were significantly different, the mean differences in OKS failed to exceed the "minimal important difference" threshold of five points [5] though the median score reached it. Although not validated for analysis by individual question, it is noteworthy that the largest differences were seen in the use of transport, kneeling and giving way, activities which rely heavily on the isokinetic quadriceps strength and extensor efficiency of the knee, where differences between CPKA and TKA are known to exist $[13,24,11]$. This ties in well with the gait data, since the most significant differences are seen in early stance, during weight acceptance and mid-stance, were the quadriceps are at their most active [30]. Whilst formal subgroup analysis is not appropriate due to demographic discrepancies, there is no obvious clustering of PROMs amongst the CPKA subgroups (Fig. 7).

Native compartment disease progression remains one of the most common reasons for revision of primary PKA, though the incidence varies between studies [10, 31, 33, 37]. In one series, of PFA revised to TKA, 56\% resulted from disease progression in the tibiofemoral compartments [27], while in another, $76 \%$ of UKA were revised for the same reason [22]. However, the 20 year series from collected by Svard, reported just $2.3 \%$ revision rate for lateral compartment progression following UKA-M in the designer centre

Table 3 Oxford Knee Scores between CPKA and TKA groups by overall score, where 48 is the best possible outcome, and by individual question

\begin{tabular}{|c|c|c|c|}
\hline & CPKA & TKA & $p$ value \\
\hline Overall OKS & $41.3 \pm 7.4$ & $37.1 \pm 5.1$ & $\mathbf{0 . 0 2}$ \\
\hline Q1. How would you describe the pain you usually have from your knee? & $2.9 \pm 1.0$ & $2.7 \pm 1.1$ & 1 \\
\hline Q2. Have you had any trouble with washing and drying yourself (all over) because of your knee? & $3.7 \pm 0.6$ & $3.8 \pm 0.4$ & 1 \\
\hline Q3. Have you had any trouble getting in or out of the car or using public transport because of your knee? & $3.3 \pm 0.9$ & $2.7 \pm 0.9$ & 0.027 \\
\hline Q4. For how long have you been able to walk before pain from your knee becomes severe? & $3.8 \pm 0.6$ & $3.6 \pm 0.7$ & 0.37 \\
\hline Q5. How painful has it been for you to stand up from a chair because of your knee? & $3.3 \pm 0.9$ & $2.9 \pm 0.8$ & 0.11 \\
\hline Q6. Have you been limping when walking because of your knee? & $3.4 \pm 1.0$ & $3.0 \pm 1.1$ & 0.46 \\
\hline Q7. Could you kneel down and get up again afterwards? & $2.7 \pm 1.3$ & $1.8 \pm 0.9$ & 0.02 \\
\hline Q8. Have you been troubled by pain from your knee in bed at night? & $3.5 \pm 0.8$ & $3.3 \pm 0.8$ & 1 \\
\hline Q9. How much has pain from your knee interfered with your usual work? & $3.5 \pm 0.9$ & $3.6 \pm 0.7$ & 1 \\
\hline Q10. Have you felt that your knee may suddenly 'give way' or let you down? & $3.8 \pm 0.7$ & $2.9 \pm 0.8$ & $<0.001$ \\
\hline Q11.Could you do the household shopping on your own? & $3.7 \pm 0.6$ & $3.8 \pm 0.5$ & 1 \\
\hline Q12. Could you walk down one flight of stairs? & $3.5 \pm 0.7$ & $3.1 \pm 0.9$ & 0.32 \\
\hline
\end{tabular}

Each question has a maximum score of 4 for best outcome. Values shown are mean with standard deviation. Significant differences are highlighted in bold 
Table 4 Euro-Qol 5D Scores between CPKA and TKA groups by overall score and by individual domain

\begin{tabular}{llll}
\hline & CPKA & TKA & $p$ value \\
\hline Overall EQ5D & $0.91 \pm 0.1$ & $0.83 \pm 0.1$ & $\mathbf{0 . 0 0 6}$ \\
Mobility & $1.4 \pm 0.7$ & $2.1 \pm 0.9$ & $\mathbf{0 . 0 0 6}$ \\
Self-care & $1.2 \pm 0.5$ & $1.2 \pm 0.4$ & 1 \\
Usual activities & $1.5 \pm 0.7$ & $2.1 \pm 0.9$ & $\mathbf{0 . 0 3}$ \\
Pain & $1.7 \pm 0.7$ & $2.2 \pm 0.7$ & $\mathbf{0 . 0 3}$ \\
Anxiety & $1.2 \pm 0.5$ & $1.3 \pm 0.6$ & 1 \\
\hline
\end{tabular}

The best possible outcome for the overall score is 1. Each domain is graded 1-5 where 1 is the best overall outcome. Values shown are mean with standard deviation. Significant differences are highlighted in bold

[36]. While at 7 years post-op, of 1000 patients whom underwent Phase 3 Oxford UKA-M, 2.5\% of them required revision for progression of $\mathrm{OA}$ in the lateral compartment [33]. In 2020, the National Institute for Health and Clinical Excellence (NICE) revised their guidelines on knee replacement, for the first time, dividing revision surgery into 'major' and 'minor' procedures, noting they have different outcomes, despite being categorised together by joint registries. NICE also observed the thresholds for revision of PKA are lower than those for revision of primary TKA, in part due to the relative technical ease of PKA to TKA procedures [16].

There is good evidence that primary PKA is safer, more cost effective and higher functioning than primary TKA [4, 17, 21, 23, 40, 41]. Removal of a well-fixed PKA can result in significant bone loss, requiring increased constraint, augments, wedges and stems $[6,25,33,42]$. The data on outcomes following revision of UKA to TKA are varied, with some studies reporting comparable rates of function and satisfaction to primary UKA [22, 29] while others report inferior outcomes compared to matched cohorts undergoing primary TKA [43]. Retention of a well-fixed, well-functioning primary PKA and targeted treatment of a newly degenerate compartment is an appealing option to PKA advocates, since the subsequent operation may be regarded as a second primary procedure. A designer series reported that the benefits of PKA also exist after staged Bi-UKA, with excellent functional outcomes, faster recovery, short hospital stays and very low complication rates [34]. This suggests that conversion to CPKA may be a good option in young people keen to avoid revision to TKA at an early age, and a safer alternative in older people or those considered high surgical risk. In terms of surgical invasiveness, the compartmental approach is a relatively minor undertaking compared to revision to TKA, and may, therefore be a conservative way of satisfying the NICE guidelines.

\section{Limitations}

This paper is limited by the lack of pre-operative gait data for either the CPKA prior to revision or the primary TKA group which would be necessary to fully assess the change in function following either procedure. However, the purpose of this study was not to prove superiority of one treatment pathway over another, rather to provide a quantitative report and insight into the function of staged CPKA, and provide context for these data through comparison to a matched primary TKA patient group. The study were powered for gait analysis as a primary outcome, while PROMs were measured as a secondary outcome.

The status of the ACL in the TKA group was not documented prospectively, and has been inferred from the appearances of the pre-operative radiographs. Therefore, whilst all patients in the TKA group were potentially suitable for PKA or primary CPKA, there may have been reasons at the time why a TKA was considered more appropriate.

The route to CPKA for the subjects was variable and the inclusion of subgroups was necessary to power the study. The heterogeneity of the groups in terms of procedure order and type primary and secondary surgery, implant type, and time to second surgery is a limitation of the study, in as much as it is underpowered for specific implant combinations, brands or revision rates. A much larger study would be required to indicate whether some subtypes demonstrate advantages over others. Procedure subgroup analysis reported here is descriptive rather than statistical, due to small numbers and skewed sex demographics. Whilst we endeavoured to include all patients whom had undergone revision to CPKA under the senior author's care, a significant number of patients were unsuitable, unable or unwilling to participate, which may have impacted upon the results. This study is underpowered to compare re-revision rates, though it is noted that four patients (5.4\%) had undergone further ipsilateral knee arthroplasty surgery, excluding them from this study. The true re-revision rates for PKA revised to CPKA are unknown. The retrospective nature of this study meant that the timepoints in post-operative evaluations was highly variable, though the groups had a similar median follow-up time. TKA subjects with only single compartment OA were included, all of whom had undergone one procedure only, whilst all CPKA subjects had undergone two procedures and some had contralateral arthroplasty, all of which introduces bias in favour of TKA. Finally, all CPKA procedures were performed by an expert high-volume partial knee arthroplasty surgeon. There is evidence that surgeons with a higher proportion of PKA practice have better outcomes $[32,34]$, therefore the results may not be generalisable to low-volume PKA surgeons at this juncture, though this may be addressed in the future through additional training for 
arthroplasty surgeons or the use of assistive technologies including robotics, the latter having proved useful at restoring near-native alignment when used to implant primary Bi-UKA [3].

\section{Clinical relevance}

Knee surgery is evolving, moving away from a "one definitive procedure" approach towards a patient-safety conscious, minor surgery "as required" strategy. This paper contributes toward the view that a compartmental approach to treatment of end-staged arthritis in a native compartment is high functioning and leads to good patient outcomes. It has the potential to support surgeons in their decision to perform primary PKA in the young, active patient, with reduced concern about subsequent surgery and the consequences of major revision. Further, it offers a safer, less invasive, bonepreserving alternative to revision to TKA in the event of progression of arthritis, which may prove particularly important in an ageing population or those who pose a high risk of medical complications.

\section{Conclusions}

This study finds that a compartmental approach to native compartment degeneration following partial knee arthroplasty results in nearer-normal gait and improved patient satisfaction compared total knee arthroplasty.

Supplementary information The online version contains supplementary material available at https://doi.org/10.1007/s00167-021-06691-9.

Acknowledgements The authors would like to thank Miss Rhiannon Lambkin and Dr. Amy Maslivec, Gait Lab Research assistants, MSk Lab, Imperial College London for their contribution to data collection.

Author contributions AG: conception of the work, design of the study, execution of the study including acquisition of the data, data analysis and interpretation, manuscript writing and editing. OD: analysis of the data, review of the manuscript. RA: interpretation of the data, review of the manuscript. JC: conception of the work, design of the study, review of the data, review and editing of the manuscript, supervision of the authors.

Funding This work was funded by the Sir Michael Uren Foundation; The Royal College of Surgeons of England and the Dunhill Medical Trust Clinical Research Fellowship. Infrastructure support was provided by the National Institute for Health Research (NIHR) Imperial Biomedical Research Centre (BRC).

\section{Declarations}

Conflict of interest The authors declare that they have no conflict of interest.
Ethical approval This projet was conducted with ethical approval from Imperial College Healthcare NHS Trust Reference Number 10/ H0807/101 and NRES Committee South Central IRAS Reference Number 136430.

Open Access This article is licensed under a Creative Commons Attribution 4.0 International License, which permits use, sharing, adaptation, distribution and reproduction in any medium or format, as long as you give appropriate credit to the original author(s) and the source, provide a link to the Creative Commons licence, and indicate if changes were made. The images or other third party material in this article are included in the article's Creative Commons licence, unless indicated otherwise in a credit line to the material. If material is not included in the article's Creative Commons licence and your intended use is not permitted by statutory regulation or exceeds the permitted use, you will need to obtain permission directly from the copyright holder. To view a copy of this licence, visit http://creativecommons.org/licenses/by/4.0/.

\section{References}

1. (2019) 16th Annual report 2019. National Joint Registry for England, Wales, Northern Ireland and the Isle of Man

2. Al-Dadah O, Hawes G, Chapman-Sheath PJ, Tice JW, Barrett DS (2020) Unicompartmental vs. segmental bicompartmental vs. total knee replacement: comparison of clinical outcomes. Knee Surg Relat Res 32:47

3. Banger MS, Johnston WD, Razii N, Doonan J, Rowe PJ, Jones BG et al (2020) Robotic arm-assisted bi-unicompartmental knee arthroplasty maintains natural knee joint anatomy compared with total knee arthroplasty: a prospective randomized controlled trial. Bone Jt J 102-b:1511-1518

4. Beard DJ, Davies LJ, Cook JA, MacLennan G, Price A, Kent S et al (2019) The clinical and cost-effectiveness of total versus partial knee replacement in patients with medial compartment osteoarthritis (TOPKAT): 5-year outcomes of a randomised controlled trial. Lancet. https://doi.org/10.1016/s0140-6736(19) 31281-4

5. Beard DJ, Harris K, Dawson J, Doll H, Murray DW, Carr AJ et al (2015) Meaningful changes for the Oxford hip and knee scores after joint replacement surgery. J Clin Epidemiol 68:73-79

6. Berend KR, George J, Lombardi AV Jr (2009) Unicompartmental knee arthroplasty to total knee arthroplasty conversion: assuring a primary outcome. Orthopedics. https://doi.org/10.3928/01477 447-20090728-32

7. Biazzo A, Silvestrini F, Manzotti A, Confalonieri N (2019) Bicompartmental (uni plus patellofemoral) versus total knee arthroplasty: a match-paired study. Musculoskelet Surg 103:63-68

8. Burger JA, Kleeblad LJ, Laas N, Pearle AD (2020) Mid-term survivorship and patient-reported outcomes of robotic-arm assisted partial knee arthroplasty. Bone Jt J 102-b:108-116

9. Chiu SS (2006) The anterior tibial translocation sign. Radiology 239:914-915

10. Crawford DA, Berend KR, Lombardi AV (2018) Management of the failed medial unicompartmental knee arthroplasty. J Am Acad Orthop Surg 26:e426-e433

11. Dandridge O, Garner A, Amis AA, Cobb JP, Arkel RJ (2021) Variation in the patellar tendon moment arm identified with an improved measurement framework. J Orthopaedic Res. https:// doi.org/10.1002/jor.25124

12. Garner A, Cobb J (2020) Combined partial knee arthroplasty. In: Rivière C, Vendittoli P-A (eds) Personalized Hip and Knee 
joint replacement. Springer International Publishing, Cham, pp 243-254. https://doi.org/10.1007/978-3-030-24243-5_21

13. Garner A, Dandridge O, Amis AA, Cobb JP, van Arkel RJ (2021) The extensor efficiency of unicompartmental, bicompartmental, and total knee arthroplasty. Bone Jt Res 10:1-9

14. Garner A, van Arkel RJ, Cobb J (2019) Classification of combined partial knee arthroplasty. Bone Jt J 101-B:922-928

15. Garner AJ, Dandridge OW, Amis AA, Cobb JP, van Arkel RJ (2021) Partial and combined partial knee arthroplasty: greater anterior-posterior stability than posterior cruciate-retaining total knee arthroplasty. J Arthroplast. https://doi.org/10.1016/j. arth.2021.06.025

16. Goodfellow JW, O'Connor JJ, Murray DW (2010) A critique of revision rate as an outcome measure: re-interpretation of knee joint registry data. J Bone Jt Surg Br 92:1628-1631

17. Gromov K, Petersen PB, Jørgensen CC, Troelsen A, Kehlet H (2020) Unicompartmental knee arthroplasty undertaken using a fast-track protocol. Bone Jt J 102-b:1167-1175

18. Haddad FS, Masri BA (2019) Compartmental arthroplasty: time for a clear nomenclature. Bone Jt J 101-b:889-890

19. Heyse TJ, Khefacha A, Cartier P (2010) UKA in combination with PFR at average 12-year follow-up. Arch Orthop Trauma Surg 130:1227-1230

20. Hof AL (1996) Scaling gait data to body size. Gait Posture $4: 222-223$

21. Jenkins C, Jackson W, Bottomley N, Price A, Murray D, Barker K (2019) Introduction of an innovative day surgery pathway for unicompartmental knee replacement: no need for early knee flexion. Physiotherapy 105:46-52

22. Jonas SC, Jermin P, Howells N, Porteous A, Murray J, Robinson J (2019) Outcome of revision UKR to TKR when compared to a matched group of TKR of same total arthroplasty lifespan. Knee 26:400-404

23. Jones G, Kotti M, Wiik A, Collins R, Brevadt M, Strachan R et al (2016) Gait comparison of unicompartmental and total knee arthroplasties with healthy controls. Bone Jt J 98-B:16-21

24. Joseph MN, Carmont MR, Tailor H, Stephen JM, Amis AA (2020) Total knee arthroplasty reduces knee extension torque in-vitro and patellofemoral arthroplasty does not. J Biomech 104:109739

25. Khan Z, Nawaz SZ, Kahane S, Esler C, Chatterji U (2013) Conversion of unicompartmental knee arthroplasty to total knee arthroplasty: the challenges and need for augments. Acta Orthop Belg 79:699-705

26. Klasan A, Parker DA, Lewis PL, Young SW (2021) Low percentage of surgeons meet the minimum recommended unicompartmental knee arthroplasty usage thresholds: analysis of 3037 Surgeons from Three National Joint Registries. Knee Surg Sports Traumatol Arthrosc. https://doi.org/10.1007/s00167-021-06437-7

27. Lewis PL, Graves SE, Cuthbert A, Parker D, Myers P (2019) What is the risk of repeat revision when patellofemoral replacement is revised to TKA? An analysis of 482 cases from a large national arthroplasty registry. Clin Orthop Relat Res 477:1402-1410

28. Liddle A, Pandit H, Judge A, Murray D (2015) Optimal usage of unicompartmental knee arthroplasty: a study of 41,986 cases from the National Joint Registry for England and Wales. Bone Jt J 97-b:1506-1511

29. Lim JBT, Pang HN, Tay KJD, Chia SL, Lo NN, Yeo SJ (2019) Clinical outcomes and patient satisfaction following revision of failed unicompartmental knee arthroplasty to total knee arthroplasty are as good as a primary total knee arthroplasty. Knee $26: 847-852$
30. Lundberg HJ, Rojas IL, Foucher KC, Wimmer MA (2016) Comparison of antagonist muscle activity during walking between total knee replacement and control subjects using unnormalized electromyography. J Arthroplast 31:1331-1339

31. Mohammad HR, Kennedy JA, Mellon SJ, Judge A, Dodd CA, Murray DW (2019) Ten-year clinical and radiographic results of 1000 cementless Oxford unicompartmental knee replacements. Knee Surg Sports Traumatol Arthrosc. https://doi.org/10.1007/ s00167-019-05544-w

32. Murray DW, Parkinson RW (2018) Usage of unicompartmental knee arthroplasty. Bone Jt J 100-b:432-435

33. Pandit H, Jenkins C, Gill HS, Barker K, Dodd CA, Murray DW (2011) Minimally invasive Oxford phase 3 unicompartmental knee replacement: results of 1000 cases. J Bone Jt Surg Br 93:198-204

34. Pandit H, Mancuso F, Jenkins C, Jackson WFM, Price AJ, Dodd CAF et al (2017) Lateral unicompartmental knee replacement for the treatment of arthritis progression after medial unicompartmental replacement. Knee Surg Sports Traumatol Arthrosc 25:669-674

35. Parratte S, Pauly V, Aubaniac JM, Argenson JN (2010) Survival of bicompartmental knee arthroplasty at 5 to 23 years. Clin Orthop Relat Res 468:64-72

36. Price AJ, Svard U (2011) A second decade lifetable survival analysis of the Oxford unicompartmental knee arthroplasty. Clin Orthop Relat Res 469:174-179

37. Scott CEH, Powell-Bowns MFR, MacDonald DJ, Simpson PM, Wade FA (2018) Revision of unicompartmental to total knee arthroplasty: does the unicompartmental implant (metal-backed vs all-polyethylene) impact the total knee arthroplasty? J Arthroplast 33:2203-2209

38. Stoddart JC, Dandridge O, Garner A, Cobb J, Arkel R (2020) The compartmental distribution of knee osteoarthritis-a systematic review and meta-analysis. Osteoarthr Cartil. https://doi.org/10. 1016/j.joca.2020.10.011

39. Studenski S, Perera S, Patel K, Rosano C, Faulkner K, Inzitari $\mathrm{M}$ et al (2011) Gait speed and survival in older adults. JAMA 305:50-58

40. Wiik AV, Manning V, Strachan RK, Amis AA, Cobb JP (2013) Unicompartmental knee arthroplasty enables near normal gait at higher speeds, unlike total knee arthroplasty. J Arthroplast 28:176-178

41. Wiik AV, Nathwani D, Akhtar A, Al-Obaidi B, Strachan R, Cobb JP (2019) The unicompartmental knee is the preferred side in individuals with both a unicompartmental and total knee arthroplasty. Knee Surg Sports Traumatol Arthrosc. https://doi.org/10. 1007/s00167-019-05814-7

42. Wilson HA, Middleton R, Abram SGF, Smith S, Alvand A, Jackson WF et al (2019) Patient relevant outcomes of unicompartmental versus total knee replacement: systematic review and meta-analysis. BMJ 364:1352. https://doi.org/10.1136/bmj.1352

43. Zuo W, Ma J, Guo W, Zhang Q, Wang W, Liu Z (2018) Comparison of the clinical outcomes of revision of failed UKAs to TKAs with primary TKAs: a systematic review and meta-analysis. Medicine (Baltim) 97:e13408

Publisher's Note Springer Nature remains neutral with regard to jurisdictional claims in published maps and institutional affiliations. 